\title{
Interlayer exchange coupling and giant magnetoresistance in $\mathrm{Fe} / \mathrm{V}$ (001) superlattices
}

\author{
A. Broddefalk, R. Mathieu and P. Nordblad \\ Department of Materials Science, Uppsala University, Box 534, SE-751 21, Uppsala, Sweden \\ P. Blomqvist and R. Wäppling \\ Department of Physics, Uppsala University, Box 530, SE-751 21, Uppsala, Sweden \\ J. Lu and E. Olsson \\ Department of Materials Science, Uppsala University, Box 534, SE-751 21, Uppsala, Sweden
}

(Dated: November 1, 2018)

\begin{abstract}
Magnetization and magnetoresistivity studies of Fe/V (001) superlattices are reported. The first giant magnetoresistance peak with respect to the vanadium and iron layer thicknesses is investigated. The interlayer antiferromagnetic coupling strength is found to show a peak at a vanadium layer thickness of 13 atomic monolayers $(\approx 20 \AA)$ with a full width at half maximum of about 2 monolayers. The antiferromagnetic coupling shows a maximum at an iron layer thickness of about 6 monolayers $(\approx 9 \AA)$ for series of superlattices with vanadium thicknesses around 13 monolayers. The magnitude of the giant magnetoresistance shows a similar variation as the antiferromagnetic coupling strength.
\end{abstract}

\section{INTRODUCTION}

The interlayer exchange coupling (IEC) of ferromagnetic layers through a non-magnetic metal has attracted a lot of attention in the last decade, in connection to the giant magnetoresistive (GMR) effect observed in antiferromagnetically coupled layers]. This interaction has been shown to oscillate between ferromagnetic (FM) and antiferromagnetic $(\mathrm{AF})$ coupling when varying the spacer layer thickness 3 . The IEC has also, both experimentally and theoretically been shown to depend on the thickness of the magnetic layers. In this case, the coupling coefficient does not necessarily change sign but may only show a varying magnitude with increasing layer thickness. Similar variations of the GMR ratio with the magnetic layer thicknesses have been reported. $\mathrm{Fe} / \mathrm{V}$ (iron/vanadium) superlattices have been shown to couple antiferromagnetically for $\mathrm{Fe}(3$ monolayers $) / \mathrm{V}(12-16$ monolayers),, and in a series of $\mathrm{Fe}(10 \AA) / \mathrm{V}\left(t_{\mathrm{V}}\right)$, oscillations were found with a maximum in the strength of the antiferromagnetic cpupling at $\mathrm{V}$ layer thicknesses of $t_{\mathrm{V}}=22,32$ and $42 \AA .0$ One atomic monolayer (ML) of a Fe/V (001) superlattice amounts to about $1.5 \AA$.

In this paper, the influence of the thickness of the $\mathrm{Fe}$ layers on the IEC and the GMR of Fe/V (001) superlattices near the first AF coupling peak $(\mathrm{V} \approx 13 \mathrm{ML})$ are examined.

\section{EXPERIMENTAL}

\section{A. Sample preparation and characterization}

The $\mathrm{Fe} / \mathrm{V}$ superlattices (SL) were grown in a three target magnetron sputtering system with a base pressure of $10^{-10}$ torr. The polished $\mathrm{MgO}$ (001) substrates $\left(10 \times 10 \times 0.5 \mathrm{~mm}^{3}\right)$ were ultrasonically precleaned in ethanol, isopropanol and acetone before they were out- gassed at $800{ }^{\circ} \mathrm{C}$ for 30 minutes. The sputtering gas was Ar with a purity of $99.9999 \%$ and the targets used were $\mathrm{Fe}(99.95 \%), \mathrm{V}(99.7 \%)$ and $\operatorname{Pd}(99.95 \%)$. The sputtering gas pressure was 5.0 mtorr and the substrate holder temperature was $400{ }^{\circ} \mathrm{C}$. The sample holder was electrically isolated from ground potential and rotated $(\sim 30$ rpm) during deposition to prevent thickness gradients. The epitaxial relationship between $\mathrm{Fe}$ and $\mathrm{MgO}(001)$ is $\mathrm{Fe}[001] \| \mathrm{MgO}$ [001] and $\mathrm{Fe}[110] \| \mathrm{MgO}$ [010]. This arrangement gives a nominal lattice mismatch of $3.5 \%$. On the substrate, Fe and V were alternately deposited by using computer-activated shutters. The layer thicknesses were monitored by the deposition time. Typical deposition rates of $\mathrm{Fe}$ and $\mathrm{V}$ were $0.65 \AA / \mathrm{s}$ and $0.45 \AA / \mathrm{s}$ respectively. The samples were capped with palladium $(\mathrm{Pd})$ to avoid oxidation. In this paper we use the nomenclature $\mathrm{Fe}(X \mathrm{ML}) / \mathrm{V}(Y \mathrm{ML})$, where $X$ and $Y$ indicate the nominal thicknesses of the $\mathrm{Fe}$ and $\mathrm{V}$ layers in atomic monolayers, respectively. The Fe thickness ranged from 3 to $13 \mathrm{ML}(X=3,5,6,9$ and $13 \mathrm{ML})$, while $\mathrm{V}$ thicknesses $(Y=11,12,13,14$ and $15 \mathrm{ML})$ were ghosen around the first AF coupling peak near $\mathrm{V} \approx 13 \mathrm{ML}$.

The structural quality of the SL were investigated by $\mathrm{XRD}$. The measurements were carried out in the lowangle region $\left(2 \theta=1-20^{\circ}\right)$ and in the high-angle region $\left(2 \theta=20-100^{\circ}\right)$. A Siemens D5000 powder diffractometer was used with the beam defined by $0.3^{\circ}$ divergence and receiving slits. For full width at half maximum (FWHM) measurements, the beam was defined by $0.05^{\circ}$ slits. The $\mathrm{Cu} \mathrm{K}_{\alpha}$ radiation was monochromatized by a secondary graphite monochromator. The SUPREX model was used in order to determine the $\mathrm{Fe} / \mathrm{V}$ interface quality 6 .

Table gives for all SL the number of atomic monolayers and repetitions, the thickness of $\mathrm{Pd}$, as well as the nominal and measured superlattice periods $\Lambda$. The nominal $\Lambda$ is estimated from $\Lambda=X \times \mathrm{a}_{F e} / 2+Y \times \mathrm{a}_{V} / 2$, where $\mathrm{a}_{F e}=2.8664 \AA$ And $\mathrm{a}_{V}=3.0274$ Aare the lattice parameters of Fe and $\mathrm{V}$ respectively. The measured $\Lambda$ value is obtained from the XRD measurements. The error on the 
superlattice period $\mid \Lambda$ (nominal) - $\Lambda$ (measured) $\mid$ is small, and amounts on average to $0.35 \AA$, i.e. less than 0.25 ML.

One of the superlattices, $\mathrm{Fe}(9 \mathrm{ML}) / \mathrm{V}(13 \mathrm{ML})$ was characterized by transmission electron microscopy (TEM). A cross-section specimen was prepared by gluing two thin film sample pieces face to face and subsequently cutting slices from the sandwich. Each slice was mechanically ground on both sides to a thickness of $100 \mu \mathrm{m}$. The slice was then dimpled to a thickness of $10 \mu \mathrm{m}$ at the specimen center whereafter the specimen was ion milled until electron transparency. The TEM analysis was carried out using both a Tecnai F30 ST field emission gun TEM operated at $300 \mathrm{kV}$ with a Gatan Imaging Filter and a Jeol 2000 FXII TEM operated at $200 \mathrm{kV}$.

\section{B. Magnetization and magnetoresistance measurements}

Hysteresis loops were recorded for all SL at $10 \mathrm{~K}$ in a Quantum Design MPMS5 Superconducting QUantum Interference Device (SQUID) magnetometer. The magnetic field was applied along the [100] and [110] directions of the Fe layers. The absolute value of the magnetization has been calculated using the total volume of the Fe layers in each SL as the magnetic volume, neglecting any influence from induced moments in the $\mathrm{V}$ layers. For the SL where the coupling was found to be ferromagnetic and an in-plane anisotropy was observed, the anisotropy constant $K$ proportional to the energy difference between the [110] and the [100] direction was deduced from the enclosed area of the two magnetization curves in the first quadrant of the magnetization vs. applied field curves. Since the field was applied in-plane, where the shape anisotropy is small, no correction of the field for demagnetization effects was done. For the SL where antiferromagnetic coupling was observed, the coupling strength was estimated from $J=\mu_{0} M_{s} H_{\text {sat }} t_{F e} / 4$, where $t_{F e}$ is the thickness of the Fe layers and $H_{\text {sat }}$ is the saturation field 0 Resistivity $\rho(H, T, \theta)$ and magnetoresistance (MR) were measured using a standard fourprobe method and a Maglab 2000 system from Oxford Instruments with a rotationary probe. The magnetoresistance was recorded in the current-in-plane (CIP) geometry. $\theta$ refers to the angle between the current and the in-plane magnetic field. The resistance was deduced for $\mathrm{H} \| \mathrm{I}(\theta=0)$ and $\mathrm{H} \perp \mathrm{I}\left(\theta=90^{\circ}\right)$ by rotating the sample and always feeding the current between the same contacts. The MR is defined as $\Delta \rho / \rho_{0}=\left(\rho_{0}-\rho_{\text {sat }}\right) / \rho_{0}$, with $\rho_{0}=\rho(H=0)$ and $\rho_{\text {sat }}=\rho\left(H=H_{\text {sat }}\right)$.

\section{RESULTS AND DISCUSSION}

A high-angle radial scan of the $\mathrm{Fe}(3 \mathrm{ML}) / \mathrm{V}(13 \mathrm{ML})$ superlattice is shown in Fig. 1. The peak at $62.5^{\circ}$ is the $\mathrm{Fe} / \mathrm{V}$ (002) Bragg peak, surrounded by five satellite peaks which originate from the superlattice periodicity. The peaks are sharp and well defined indicating a high structural quality of the sample. The structural coherence length $(\zeta)$ in the growth direction can be estimated from the linewidth of the Bragg peak using $\zeta=1 / \Delta q$, where $\Delta q$ is the linewidth (FWHM in $\AA^{-1}$ ) in the radial direction, $q=2 \sin \theta / \lambda$ is the scattering vector and $\theta$ is the angle of the incident and the diffracted $\mathrm{x}$-rays with respect to the sample. An out-of-plane structural coherence length of about 400 Åwas obtained for the Fe(3 ML)/V(13 ML) superlattice. No other peaks than those seen in Fig. 1 were detected in the range $2 \theta=20-100^{\circ}$ except reflections from the substrate. Furthermore, a texture scan performed on the $\mathrm{Fe}(3 \mathrm{ML}) / \mathrm{V}(13 \mathrm{ML})$ superlattice showed four (220) peaks separated by $90^{\circ}$ indicating a single-crystalline superlattice.

The SUPREX model was used to determine the $\mathrm{Fe} / \mathrm{V}$ interface roughness. The specular component of the lowangle x-ray diffraction data from the $\mathrm{Fe}(3 \mathrm{ML}) / \mathrm{V}(13 \mathrm{ML})$ superlattice in the range $2 \theta=2-8^{\circ}$ is shown in Fig. 2. The result from the fitting procedure is also shown in the figure where two distinct superlattice satellites are clearly visible. The decrease in intensity of the satellites corresponds to an average interface roughness of about two atomic monolayers $(\sim 3 \AA)$. Furthermore, the results from the fit also indicate that the Fe-on- $\mathrm{V}$ (Fe deposited on $\mathrm{V}$ ) interfaces have a somewhat larger roughness than the V-on-Fe interfaces, a result which is consistent with a recent Mössbauer investigation of the $\mathrm{Fe} / \mathrm{V}$ interfaces $\mathrm{B}$. It should be pointed out that x-ray diffraction furnish structural information averaged over length scales corresponding to the coherence length of one photon. In the $\mathrm{x}$ ray diffraction setup that was used, the effective in-plane coherence length of the radiation at low angles is limited by the spectral resolution $\Delta \lambda / \lambda$, to about $1000 \AA$. This means that we are measuring random interface roughness as well as correlated roughness induced by the substrate. Reflection high energy electron diffraction (RHEED) patterns of the Fe and $\mathrm{V}$ surfaces indicate a two-dimensional layer by layer growth of both materials.

The TEM micrograph in Fig. 3 shows a cross-section of the $\mathrm{Fe}(9 \mathrm{ML}) / \mathrm{V}(13 \mathrm{ML})$ superlattice. The superlattice exhibits flat layers, with no significant thickness fluctuations or waviness. Superlattice satellite reflections are observed around the $\mathrm{Fe} / \mathrm{V}$ (002) diffraction spot in the selected area electron diffraction (SAED) pattern shown in the figure. It is also evident that the specimen is singlecrystalline and the epitaxial relationship between the superlattice and the substrate is $\mathrm{Fe} / \mathrm{V}[001] \| \mathrm{MgO}$ [001] and $\mathrm{Fe} / \mathrm{V}[110] \| \mathrm{MgO}$ [010], as discussed above. A detailed TEM investigation of the interface quality will be further performed.

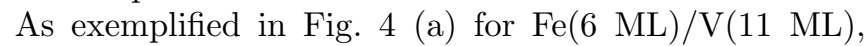
the SL with a V layer thickness of 11 monolayers, all showed four-fold in-plane anisotropy, with [100] as the easy axis, except for $\mathrm{Fe}(3 \mathrm{ML}) / \mathrm{V}(11 \mathrm{ML})$, which appeared isotropic in-plane. This sample saturated at very low fields, which also excludes antiferromagnetic 
(AF) coupling. The difference in magnetocrystalline anisotropy energy $\left(E_{a}\right)$ between the [110] and the [100] directions $\Delta E_{a}=E_{a}[110]-E_{a}[100]$, proportional to the anisotropy constant $K$, increased with the thickness of the magnetic layers. $K$, however, does not exhibit the $K=K_{v}+2 K_{s} / t_{F e}$ dependence $\left(t_{F e}\right.$ is the thickness of the magnetic layers, $K_{v}$ the volume and $K_{s}$ the surface coefficient of the magnetocrystalline anisotropy) which is expected if all SL are equally strained (cf. insert (a)). The observed deviations from such a behavior are probably due to the magnetoelastic coupling since the Fe layers are strained to accommodate a common in-plane lattice parameter with the $\mathrm{V}$ layers.

$\mathrm{Fe}(3,6$ and $9 \mathrm{ML}) / \mathrm{V}(13 \mathrm{ML})$ are magnetically isotropic in-plane, but rather large fields are required to reach saturation, implying that the Fe layers are antifer-

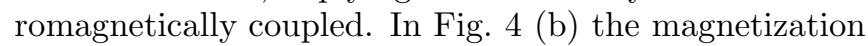
curve for the $\mathrm{Fe}(6 \mathrm{ML}) / \mathrm{V}(13 \mathrm{ML})$ is plotted. The saturation field is considerably larger for the isotropic $\mathrm{V}(13$ ML) SL than for the V(11 ML) SL measured along the hard [110] direction. For a larger Fe thickness, Fe(13 $\mathrm{ML}) / \mathrm{V}(13 \mathrm{ML})$, the superlattice shows a restored fourfold in-plane anisotropy and the magnetization curves for this sample is very similar to the corresponding curve for the Fe(13 ML)/V(11 ML) SL. This shows that magnetization wise, the AF interlayer coupling has become unresolvably weak for Fe layers in the thickness range 9-13 ML; as seen in the insert (b), the saturation field of the AF coupled SL drastically decreases when increasing the amount of Fe in the superlattice.

The magnetic field dependence of the normalized resistivity $\left(\rho / \rho_{\text {sat }}\right.$ or $\left.\rho / \rho_{0}\right)$ for the $\mathrm{Fe}(3,6$ and $9 \mathrm{ML}) / \mathrm{V}(13$ ML) SL, as well as for the Fe(13 ML)/V(11 ML) sample are shown in Fig. 5. GMR is observed for the first three samples, superposed with an increasing anisotropic magnetoresistance (AMR) component of same order of magnitude as for the corresponding sample in the $\mathrm{V}(11$ ML) series. Fe(13 ML)/V(13 ML), on the other hand, displays like Fe(13 ML)/V(11 ML) only AMR features. The saturation fields derived from the GMR curves agree with the corresponding values derived from the magnetization measurements for the SL with thin Fe layers, whereas for the $\mathrm{Fe}(9 \mathrm{ML}) / \mathrm{V}(13 \mathrm{ML})$, a saturation field is clearly seen in the MR behavior but is not resolvable in the magnetization curves. The corresponding value of $\Delta \rho / \rho_{0}$ for the current $\mathrm{Fe}(3 \mathrm{ML}) / \mathrm{V}(13 \mathrm{ML})$ is lower than a previously reported value (3\% compared to $7 \%$ ), which could be attributed to the difference in thickness of the capping layer of Pd used, (100 $\AA$ compared to 30 $\AA$ in the earlier study) as will be discussed below. This changes the amount of current going through the superlattice, which in turn affects the MR ratio. In addition, changing the thicknesses of the capping layer may also change the coupling strength.12 This effect is expected to be of less importance in our case.

The SL in the V(11 ML) series show only AMR, as shown in the insert of Fig. 局. The size of $\Delta \rho / \rho_{0}$ is increasing with increasing Fe layer thickness, as may be ex- pected from the increased magnetic layer thickness and increasing magnetocrystalline anisotropy.

In Fig. 6 (a) the AF interlayer coupling strength is plotted vs. the number of $\mathrm{V}$ monolayers for SL with 3, 5 and $9 \mathrm{ML}$ of Fe. The three series of Fe/V SL show a maximum of the AF coupling strength at a $\mathrm{V}$ thickness of about 13 ML. In Fig. 6 (b), the AF coupling strength is plotted vs. the Fe layer thickness for series of SL with $\mathrm{V}$ thicknesses of 12, 13 and $14 \mathrm{ML}$. The AF coupling strength is weaker at a $\mathrm{V}$ thickness of $12 \mathrm{ML}$, but all series of $\mathrm{Fe} / \mathrm{V}$ SL show similar trends for the dependence of the coupling strength on the Fe thickness, a shallow maximum at about $6 \mathrm{ML}$ of Fe may be estimated for all $\mathrm{V}$ thicknesses.

The MR values of SL in the V(11 ML) and V(13 ML) series are displayed in Fig. I7 (a). It is worth to note that the two Fe(13 ML) SL show an almost identical behavior only exhibiting an AMR effect. The GMR for the V(13 ML) series show a maximum of about $5 \%$ at an Fe layer thickness of about $6 \mathrm{ML}$.

The $\mathrm{Fe} / \mathrm{V}$ SL included in this study show a zero magnetic field resistivity ratio between $300 \mathrm{~K}$ and $10 \mathrm{~K}$ $[\rho(300 K) / \rho(10 K)]$ of about 2 (see inset of Fig. 7). We have observed that the resistivity ratio and the magnitude of the measured GMR ratio show a considerable covariation. A larger resistivity ratio yields a lower GMR value for nominally similar Fe/V SL. One obvious reason behind this behavior is as mentioned above a difference in thickness of the Pd capping layer that we always grow to protect the Fe/V SL from oxidation. Fig. I(b) shows the effect of this layer on the (magneto)resistivity for an $\mathrm{Fe}(5 \mathrm{ML}) / \mathrm{V}(13 \mathrm{ML}) \mathrm{SL}$. Without Pd, or for a small thickness of $\mathrm{Pd}$, the resistivity ratio between room and helium temperatures amounts to $\sim 1.3$. It increases by more than a factor of two for $\sim 100$ Aof Pd. At the same time the magnitude of the GMR effect drops from $\sim 8-9 \%$ to $\sim 3 \%$. Because of this large variation, we consider in Fig. I(a) only the SL showing similar resistivity ratios, as seen in the insert; the GMR values in this plot are thus directly comparable between each other. Of course, differences in the crystalline quality of the films and interfaces may influence the GMR magnitude 13

\section{CONCLUSIONS}

The interlayer exchange coupling of Fe/V (001) superlattices shows a first antiferromagnetic maximum at a $\mathrm{V}$ thickness of about $13 \mathrm{ML}$. At a V thickness of $13 \mathrm{ML}$, the coupling has a maximum strength at an Fe layer thickness of about 6 ML. The magnetoresistance shows GMR effects for the AF coupled SL with a maximum magnitude at the Fe thickness where the AF coupling is largest. It is noted that nominally similar Fe/V SL with similar magnitude of the AF coupling can show remarkably different GMR ratios. One simple explanation for this behavior is shunting through a metallic capping layer of different thickness. Measurements on a series of $\mathrm{Fe} / \mathrm{V}$ 
superlattices with a $\mathrm{V}$ thickness of $11 \mathrm{ML}$ showed four fold in-plane anisotropy and only anisotropic magnetoresistance for all Fe layer thicknesses. An AMR of the same magnitude was seen superposed on the GMR effect for the series of SL with a V thickness of $13 \mathrm{ML}$.

\section{Acknowledgments}

Financial support from the Swedish natural Science Research Council (NFR).
1 B. Dieny, J. Magn. Magn. Mater. 136, 335 (1994).

2 P.M. Levy, Sol. State Phys. 47, 367 (1994).

3 S. S. P. Parkin, N. More, K. P. Roche, Phys. Rev. Lett. 64, 2304 (1990).

4 P. Bruno, Phys. Rev. B 52, 411 (1995); P. J. H. Bloemen, M. T. Johnson, M. T. H. van de Vorst, R. Coehoorn, J. J. de Vries, R. Jungblut, J. aan de Stegge, A. Reinders and W. J. M. de Jonge, Phys. Rev. Lett. 72, 764 (1994); P. Lang, L. Nordström, K. Wildeberger, R. Zeller, P. H. Dedrichs, T. Hoshino Phys. Rev. B 53, 9092 (1996); P. Bruno, J. Phys.: Condens. Matter 11, 9403 (1999).

${ }^{5}$ P. Granberg, P. Isberg, E. B. Svedberg, B. Hjörvarsson, P. Nordblad and R. Wäppling, J. Magn. Magn. Mater. 186, 154 (1998).

${ }^{6}$ E. E. Fullerton, I. K. Schuller, H. Vanderstraeten and Y. Bruynseraede, Phys. Rev. B 45, 9292 (1992).

7 M. M. Schwickert, R. Coehorn, M. A. Tomaz, E. Mayo, D. Lederman, W. L. O'Brien, Tao Lin and G. R. Harp, Phys. Rev. B 57, 13681 (1998).

8 B. Kalska, P. Blomqvist, L. Häggström and R. Wäppling, Europhys. Lett. 53, 395-400 (2001).

9 É. du Trémolet de Lacheisserie, Ann. Phys. 5, 267 (1970); É. du Trémolet de Lacheisserie, Phys. Rev. B 51, 15925 (1995).

10 P. Granberg, P. Nordblad, P. Isberg, B. Hjörvarsson and R. Wäppling, Phys. Rev. B 54, 1199 (1996).

11 A. Broddefalk, P. Nordblad, P. Blomqvist, P. Isberg, R. Wäppling, O. Le Bacq and O. Eriksson, to appear in J. Magn. Magn. Mater.

12 A. Bounouh, P. Beauvillain, P. Bruno, C. Chappert, R. Mégy and P. Veillet, Europhys. Lett. 33, 315 (1996).

13 A. Moser, U. Krey, A. Paintner and B. Zellermann, J. Magn. Magn. Mater. 183, 272 (1998).

TABLE I: Data on the $\mathrm{Fe}(X \mathrm{ML}) / \mathrm{V}(Y$ ML) superlattices: Number of atomic monolayers and repetitions, Pd thickness, as well as nominal and measured superlattice period $\Lambda$.

\begin{tabular}{|c|c|c|c|}
\hline Superlattice & $\operatorname{Pd}(\AA)$ & Nominal $\Lambda(\AA)$ & Measured $\Lambda(\AA)$ \\
\hline $3 / 11 \times 30$ & 100 & 20.95 & 20.80 \\
\hline $3 / 12 \times 30$ & 100 & 22.46 & 22.95 \\
\hline $3 / 13 \times 30$ & 100 & 23.98 & 24.30 \\
\hline $3 / 14 \times 30$ & 100 & 25.49 & 25.85 \\
\hline $3 / 15 \times 30$ & 100 & 27.00 & 27.65 \\
\hline $5 / 12 \times 30$ & 100 & 25.33 & 25.30 \\
\hline $5 / 13 \times 30$ & 100 & 26.84 & 27.00 \\
\hline $5 / 13 \times 30$ & 20 & 26.84 & 26.50 \\
\hline $5 / 13 \times 30$ & 0 & 26.84 & 26.60 \\
\hline $5 / 14 \times 30$ & 100 & 28.36 & 28.25 \\
\hline $6 / 11 \times 30$ & 100 & 25.25 & 25.20 \\
\hline $6 / 13 \times 30$ & 100 & 28.28 & 28.30 \\
\hline $9 / 11 \times 30$ & 100 & 29.55 & 28.35 \\
\hline $9 / 12 \times 30$ & 100 & 31.06 & 31.05 \\
\hline $9 / 13 \times 30$ & 100 & 32.58 & 32.80 \\
\hline $9 / 14 \times 30$ & 100 & 34.09 & 34.15 \\
\hline $13 / 11 \times 30$ & 100 & 35.28 & 36.70 \\
\hline
\end{tabular}

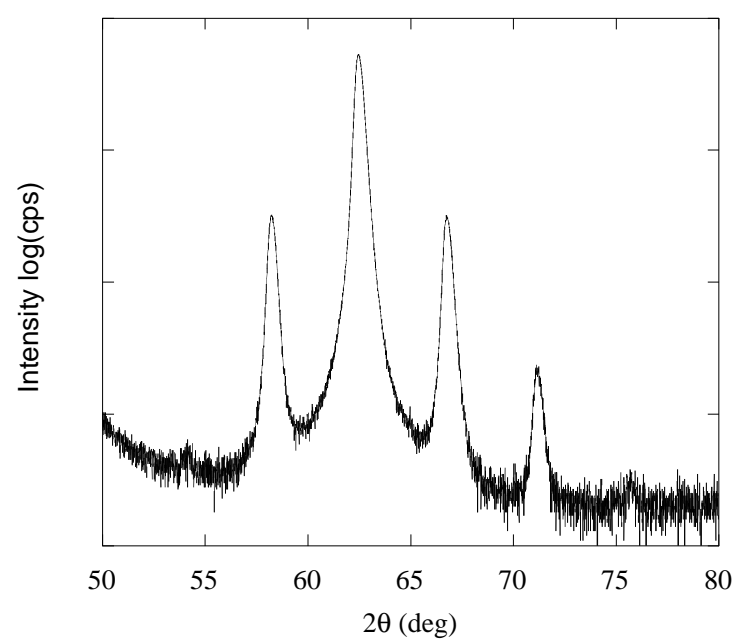

FIG. 1: High-angle x-ray diffraction scan from the $\mathrm{Fe}(3$ ML)/V(13 ML) superlattice. 


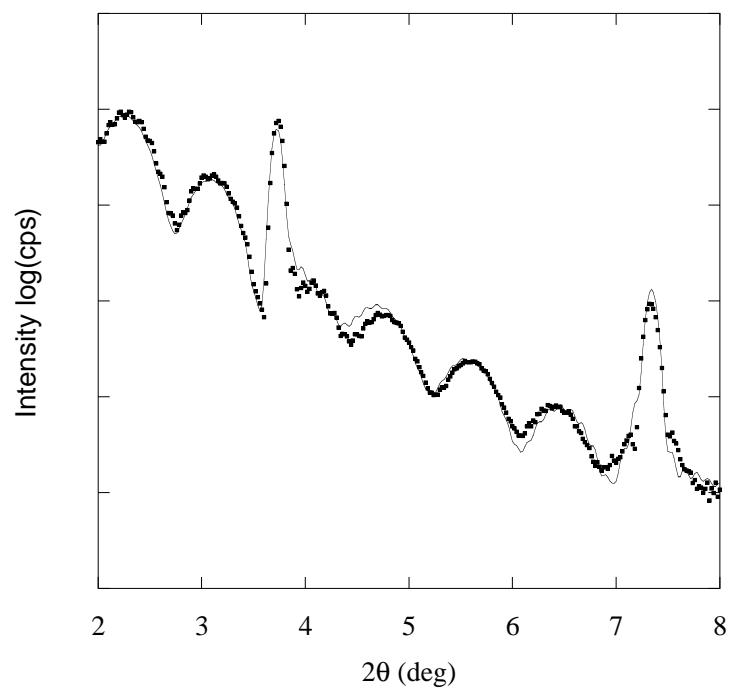

FIG. 2: Low-angle x-ray diffraction scan (specular component) for the $\mathrm{Fe}(3 \mathrm{ML}) / \mathrm{V}(13 \mathrm{ML})$ superlattice. The solid line is the fit to the measured data (filled squares).

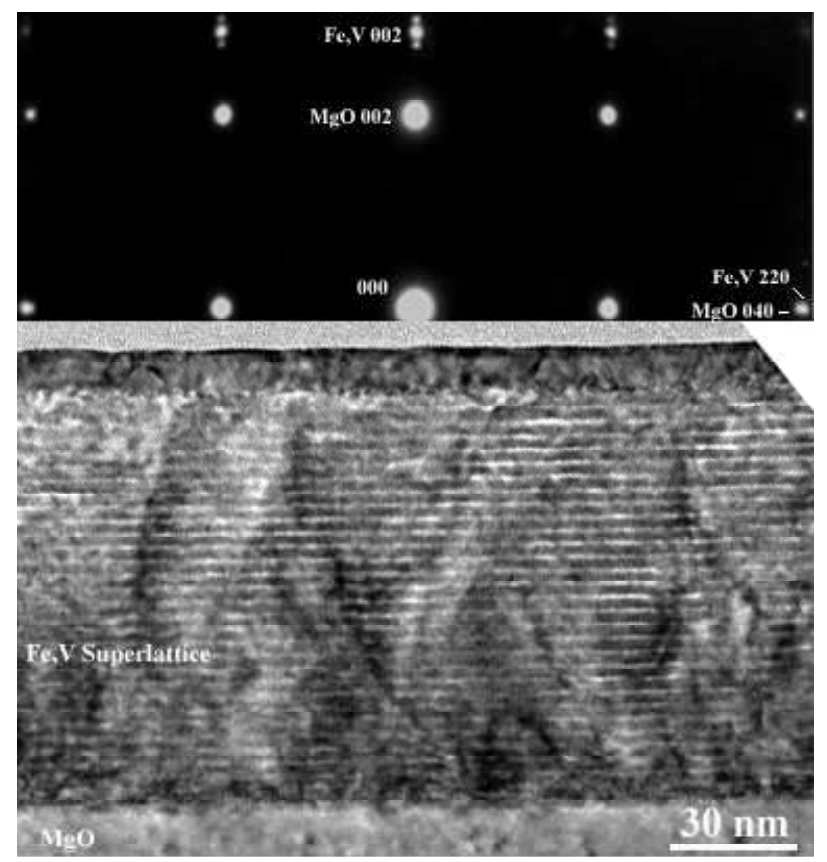

FIG. 3: SAED pattern (top) and TEM micrograph (bottom) of the $\mathrm{Fe}(9 \mathrm{ML}) / \mathrm{V}(13 \mathrm{ML})$ superlattice. 

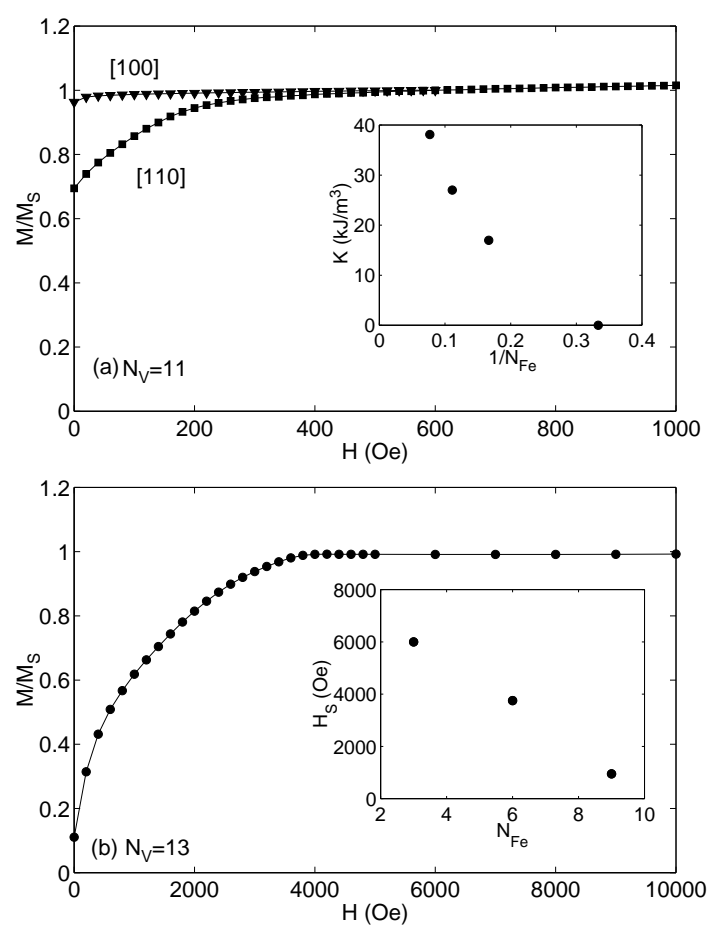

FIG. 4: Magnetization vs. magnetic field for (a) $\mathrm{Fe}(6$ $\mathrm{ML}) / \mathrm{V}(11 \mathrm{ML})$ and (b) $\mathrm{Fe}(6 \mathrm{ML}) / \mathrm{V}(13 \mathrm{ML}) ; T=10 \mathrm{~K}$. For the FM coupled SL (insert (a)), the variation of the anisotropy constant $K$ with the inverse of the Fe thickness is included. In the AF case (insert (b)), the variation of the saturation field is added.

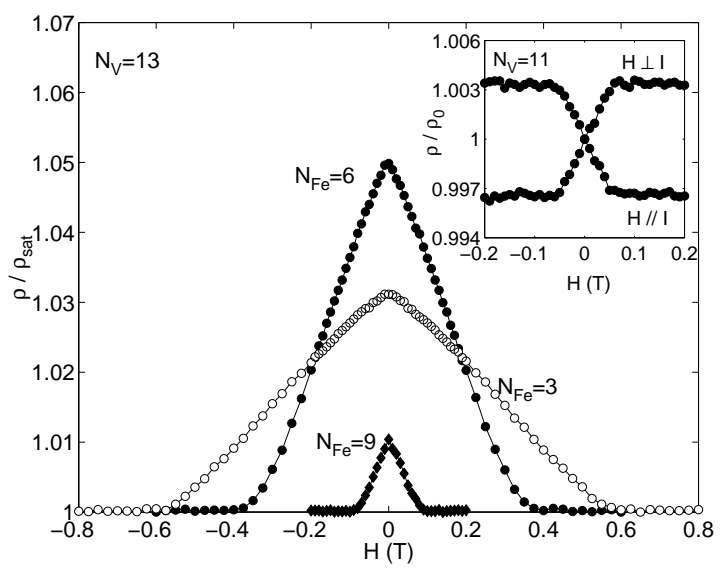

FIG. 5: Magnetoresistance curves for the AF coupled SL (main frame) and for one of the FM coupled SL (insert). 

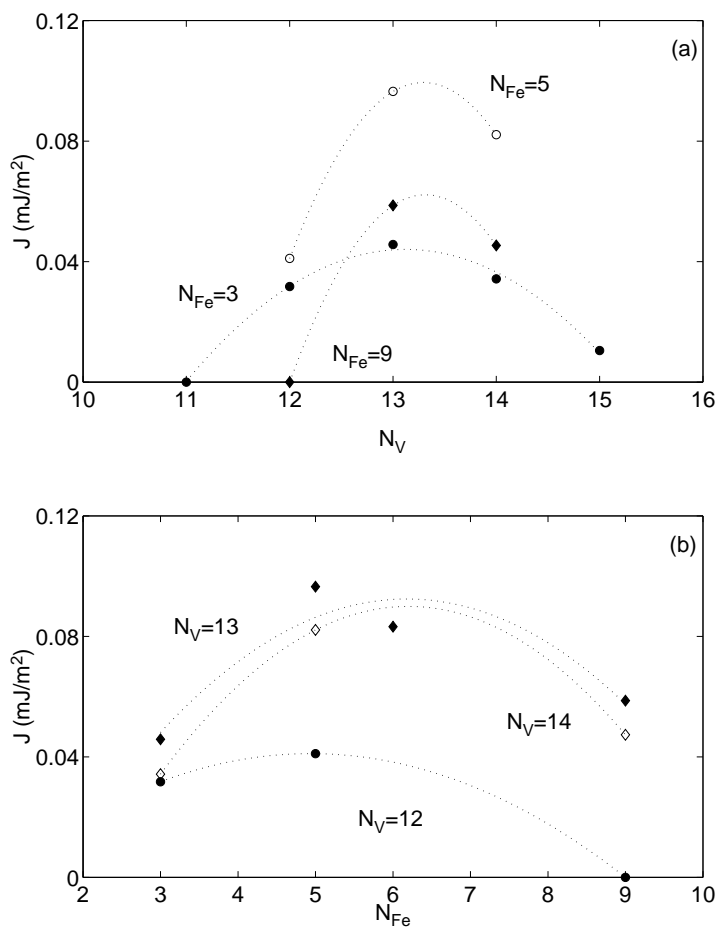

FIG. 6: Coupling strength of the antiferromagnetically coupled SL for (a) varying $\mathrm{V}$ thickness and (b) varying Fe thickness. The dotted lines are a guide to the eyes. 

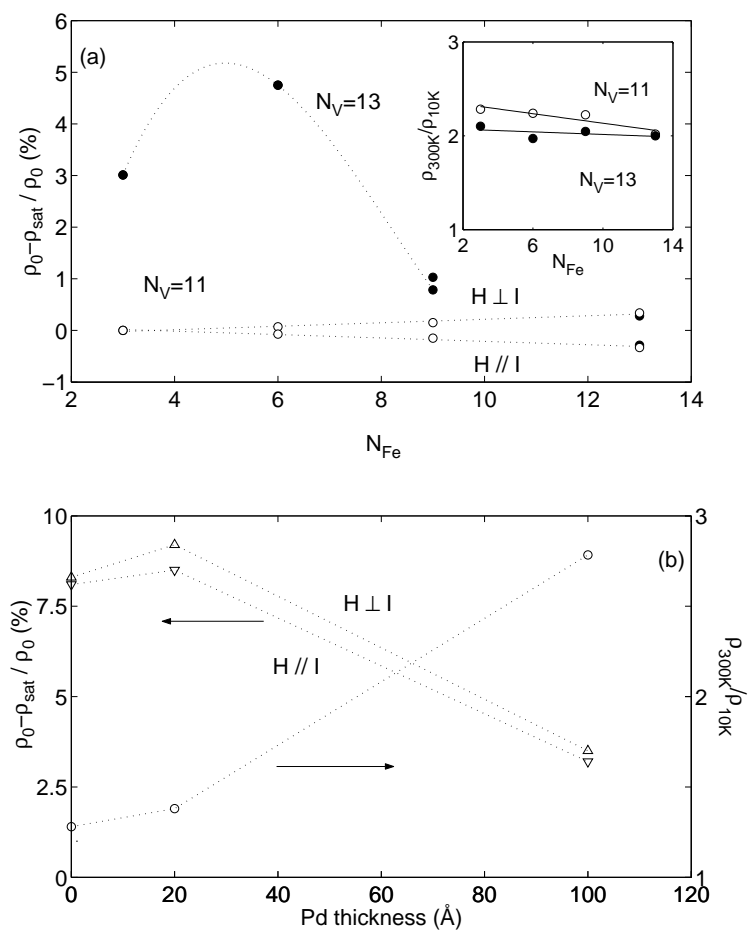

FIG. 7: (a) Magnitude of the GMR and AMR vs. $\mathrm{N}_{F e}$ for all SL. The insert shows the corresponding $\rho_{300 K} / \rho_{10 K}$ resistivity ratio. (b) illustrates the contribution of the $\mathrm{Pd}$ capping layer to the (magneto)resistivity, showing the GMR (right) and resistivity (left) ratios of $\mathrm{Fe}(5 \mathrm{ML}) / \mathrm{V}(13 \mathrm{ML}) \mathrm{SL}$ with respectively 0, 20, and 100 Åof $\mathrm{Pd}$. The dotted lines are a guide to the eyes. 
This figure "TEM.gif" is available in "gif" format from: http://arxiv.org/ps/cond-mat/0111255v1 